\title{
Enhancing Network Lifetime in Wireless Sensor Networks using Adaptive Threshold based Clustering
}

\author{
Ankit Modi \\ M.Tech Scholar \\ Lord Krishna College of Technology Indore, M.P.
}

\author{
Sanjay Thakur, $\mathrm{PhD}$ \\ Principal \\ Lord Krishna College of Technology Indore, M.P.
}

\begin{abstract}
As IoT (Internet of things) has progressed at a massive pace, the clustering algorithms that are implemented for the wireless sensor networks have started to attain enormous importance. The wireless sensor network collected together mainly constitutes an IoT framework. A Wireless Sensor Network (WSN) is composed of a number of sensor nodes that are connected to each other. An optimized energy efficient routing protocol for the enhancement of network lifetime in Wireless Sensor Networks is proposed by this paper. The chief use of the protocol used lies in the fact that at the start of each iteration, it modifies the cluster size along with the cluster head in a dynamic manner. Also taken into account are the heterogeneous and non heterogeneous networks. A threshold based approach is also made use of in order to decrease the number of total transmissions that greatly reduces the consumption of energy. A concept of delay time is put forth for re-transmission of the data considering case of not exceeding the transmission threshold limit. This sort of secures the algorithm to a great extent. The results achieved show that the system proposed yields higher network lifetime in comparison to the previously existing methods.
\end{abstract}

\section{General Terms}

Clustering, Cluster Head, Network Lifetime

\section{Keywords}

Internet of Things (IoT), Wireless Sensor Networks (WSN), Clustering, Routing, Network Lifetime

\section{INTRODUCTION}

Wireless Sensor Network can be referred as a cumulative set of many sensor nodes. The main role of the sensor nodes is to gather sensory information and data and also it performs interaction with other nodes within the network. A sensor node consists of a microcontroller, transceiver, external memory and sensors. It is of paramount necessity to enhance and improve the network lifetime of the Wireless Sensor Networks as when the sensor nodes are dead; it leads to the entre network going down. This requires that any ongoing processes to be stopped till the energy resources of the network are fully replenished to be able to function smoothly. WSNs are applied extensively in various areas such as defence, automation, robotics, chemical industries, maritime research etc. The process of clustering is utilized to enhance the capacity and lifetime of the networks.[3] Different network protocols have varied pros and cons, but mainly designing of networks demands fulfilment of two general conditions:

1) There must be reduced energy consumption per iteration of data transmission.

2) Reliability in gathering and transmitting data
The WSN is comprised of two way communication type, one being among the cluster head and base station and other being between clusters. This case is illustrated in the figure below

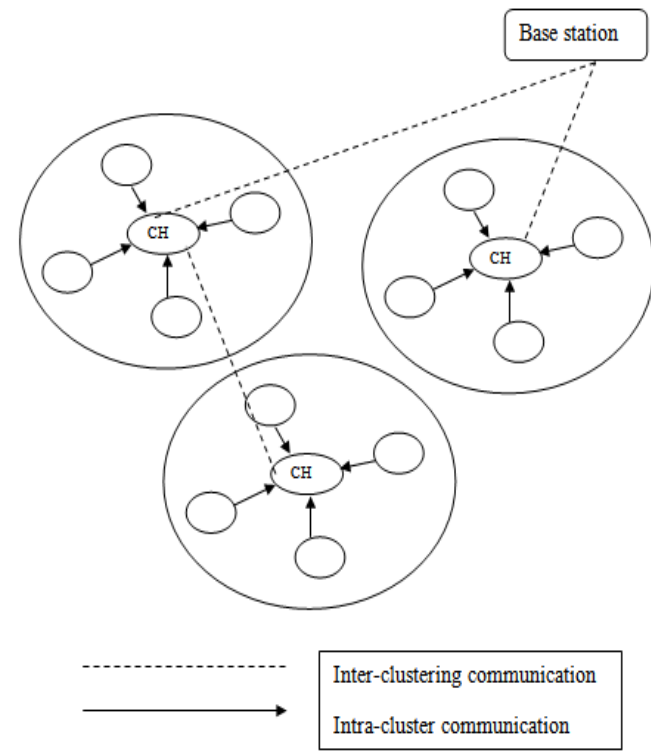

Fig 1:A clustered WSN Communication

\section{CHALLENGES IN CLUSTERING APPROACH}

The chief challenges that are identified in a cluster based routing in WSNs are as follows:

1) To decide efficiently the configuration of the set of clusters in each and every iteration of transmission of data that can help in reduced energy consumption.

2) Trying to reduce the average energy decay of nodes in relation to the iterations.

3) To avoid the nodes that are dead with respect to iterations with the aim of increasing the Quality of Service (QoS) of the system proposed.

4) To improve and enhance the network lifetime of the system.

When the cluster size or cluster head is fixed, that results in large consumption of energy and consequently gives decreased network lifetime. [6] Therefore the clustering and routing algorithm require being adaptive in nature. So that it is adaptive to search for cluster heads in all the iteration of the process Apart from the challenges aforementioned, few other areas that need to be taken into consideration while designing clustering algorithms are: 
- Cluster establishment: The $\mathrm{CH}$ determination and cluster creation methods have to produce good quality clusters that have good conceivable features like proper adjustment etc. Also it is required that the clusters are designed and arranged such that they are able to secure the total number of message information exchanged and also the predictability of the net time needs to remain the same and consistent which must be flexible. This served as one of the most difficult challenges to be addressed.

- Dependence on Application: Whilst planning of the clustering and conventions formulation for the WSNs, the robustness of the application must be of great importance and the network protocols must be designed such that they adhere and adjust to the different variations that may get introduced in the process further As it is observed in the traditionally existing networks information security is of pivotal importance in the Wireless Sensor Networks. So the ability of the WSN to save the secure communication and transmission of data is also an important parameter to be seen.

- Synchronization: Transmission methods that are slotted in nature like the TDMA et al. TDMA allows the network nodes to schedule intervals of sleep in a consistent manner in order minimize the consumption of energy. So such synchronization is also necessary and forms a useful ground for the efficient functioning of the cluster nodes. [7]

- Aggregation of Data: As this method makes improvement in the energy consumption process so it is rendered as one of the most viable methods and challenging areas in terms of data aggregation approach. This approach must also satisfy the standard of flexibility and reusability in an easy way. It can be further advanced to a much more developed system of functions. [8]

\section{PROPOSED APPROACH}

The approach proposed here is based on two main components v.i.z. adaptive clustering and a threshold based approach for data transmission.

1) Initial stage consists of deciding the spatial dimensions ( $x$, $\mathrm{y})$, and the number of nodes be $(\mathrm{N})$, let initial energy of nodes (E), optimal election probability $\left(\mathrm{p}_{\mathrm{opt}}\right)$, threshold one $\left(\mathrm{H}_{1}\right)$ and threshold two $\left(\mathrm{S}_{2}\right)$.

2) Then, the cluster $(\mathrm{CH})$ has to be decided in a random way. Simultaneously, the cluster head is chosen and decided upon using the measure of differential count amidst the energy of the nodes and the average energy of the nodes. The average energy of the nodes on all iterations $(\mathrm{K})$ is calculated as:

$\bar{E}(K)=\frac{1}{M} \sum_{i=1}^{K} E_{i}(K)$

Here, it is considered that WSN contains M number of nodes.

3) Now thirdly the cluster head for all iterations is decided based on the following relation:

$\operatorname{Enr}_{i}(\mathrm{t})=\frac{E_{\text {initial }}-E_{i}(t)}{k-1}$

Here Enr signifies the residual energy that is there with a node which is representing a function of time. As the number of iterations (k) will increase, the residual energy along with the average energy of the nodes shall decrease monotonically during the process of transfer of data: The threshold of probability for a node turning into a cluster node:[3]

$T\left(S_{i}\right)=\left\{\begin{array}{cc}\frac{p_{i}}{1-p_{i}\left(k m o d \frac{1}{p_{i}}\right)} & \text { if } s \in S \\ 0 & \text { otherwise }\end{array}\right.$

Here,

$\mathrm{S}$ signifies the set of nodes that are able to become a cluster head at any given iteration ' $\mathrm{k}$ '.

$\mathrm{p}_{\mathrm{i}}$ denotes the individual probability of a node turning into a cluster head of the $\mathrm{n}$ nodes of the network. It is given by:

$$
\operatorname{prob}_{\mathrm{i}}=1 / \mathrm{M}_{\mathrm{i}}
$$

The cumulative probability distribution function for all nodes turning into cluster heads at some iteration can is put as given below:

$\sum_{i=1}^{M} P_{i}=\sum_{i=1}^{k} P_{\text {opt }} \frac{E_{i}(k)}{\bar{E}(k)}=\sum_{i=1}^{N} \frac{E_{i}(k)}{\bar{E}(k)}=M p_{\text {opt }}$

It is observed with clarity that the gradient of energy is given by:

$\frac{d E_{i}}{d E_{r}}=g$

4) If there exist a case wherein there are heterogeneous nodes whose initial energies are inconsistent, then the probability of a node to be a cluster head is given by

$p_{\text {advanced }}=\frac{P_{\text {opt }}}{1+a m}, P_{n r m}=\frac{P_{o p t}(1+a)}{1+a m}$

Here,

Padvanced refers to the probability of an advanced node to be considered a cluster head.

Pnrm refers to the probability of a normal node to be adjudged a cluster head.

' $a$ ' and ' $m$ ' are parameters based on which the weighted probabilities are calculated.

Hence the overall combined probability of any node (normal or advanced) turning into a conceivable cluster head is given as:

$\left(P_{i}\right)=$

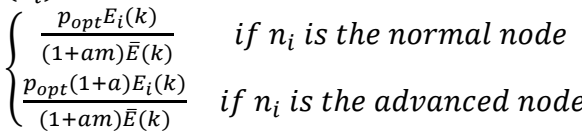

5) Many a times the WSNs sensor work in such an environment where the normal values are slow to reach on time due to the problem of time lag and keep on being stabilized in a specific range of value. As a result, good amount of energy gets consumed in transmission of the data till it reaches the ideal value of that range of data in its area. So here comes the need of a hard threshold value $\left(\mathbf{H}_{\mathrm{T}}\right)$ which is decided beforehand so that no data transmission takes place prior to it.

\section{Decide Threshold 1}

If somehow the parameter value happens to reach and touch upon the ideal periphery of operation and stays in that state due to regulation of the system controlled attributes. Then sending the data at every interval that is fixed won't produce enough information but on the contrary would lead to power 
consumption unnecessarily. This concept forms a part of information theory and is gives as below :

$I=\log _{2}\left(1 / P_{i}\right)(9)$

Here,

I illustrate the information contained in an event

$\mathrm{P}$ refers to the probability of occurrence of the event.

It is worth observing that the saturation of sensor values to a particular level does not give much information.

Hence decide Threshold_2 (T2)

Delay Time $\left(\mathbf{T}_{\mathrm{d}}\right)$ : It can also be a possibility that the data transmission from the head cluster to the base station breaks and stops in middle if the value doesn't exceed the soft threshold. Moreover a long period of no transmission of data can lead to threats concerning security and also makes the process quite unreliable. Hence the delay time is introduced so as it compensate for the same. Even if the soft threshold is not exceeded, the data has to be transmitted again after exceeding the delay time.

Hence deicide Delay Time

6) The average energy of nodes is calculated iteratively and also the value of average network lifetime in the same manner. The network lifetime is evaluated based on the number of rounds making it possible to send with relation to the variations in the nodes number within the network.

\section{RESULTS}

The simulation of the designed system has been done on MATLAB 2017a with the network parameters as given underneath:

WSN Area $=100 \mathrm{~m} \times 100 \mathrm{~m}$

Nodes $(n)=400$

Popt $=0.2$

Initial Energy of Nodes $\left(\mathrm{E}_{\mathrm{i}}\right)=0.1$ Joule

Threshold_1 $=200$

Threshold_2 = 2

Distance of base station $(\mathrm{d})=200 \mathrm{~m}$

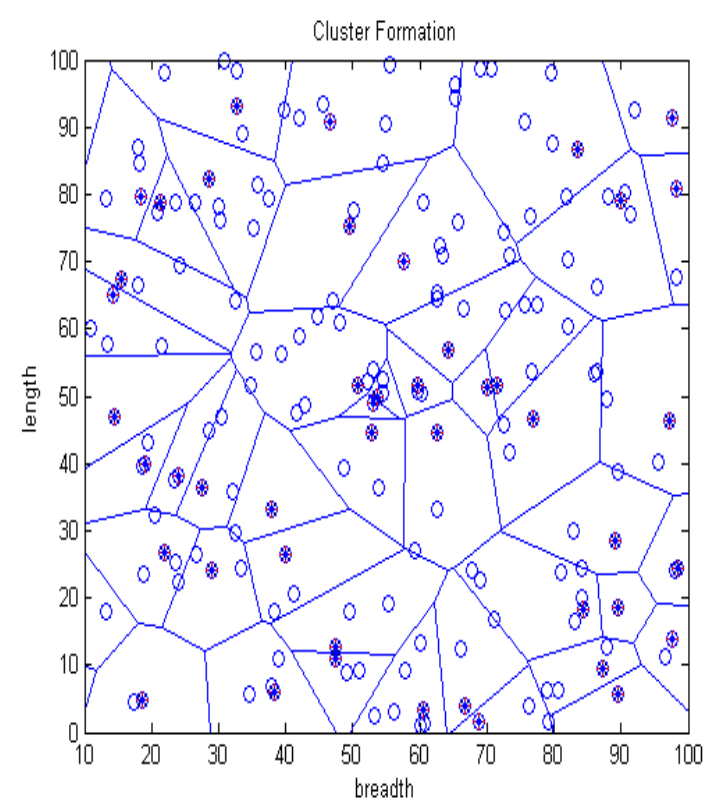

Fig 2:Formation of Clusters

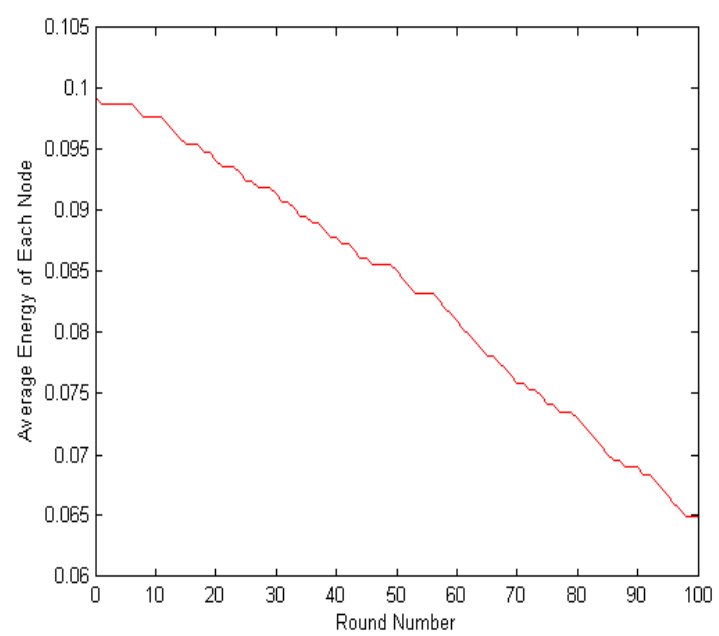

Fig 3: Nodes Average Energy w.r.t number of rounds

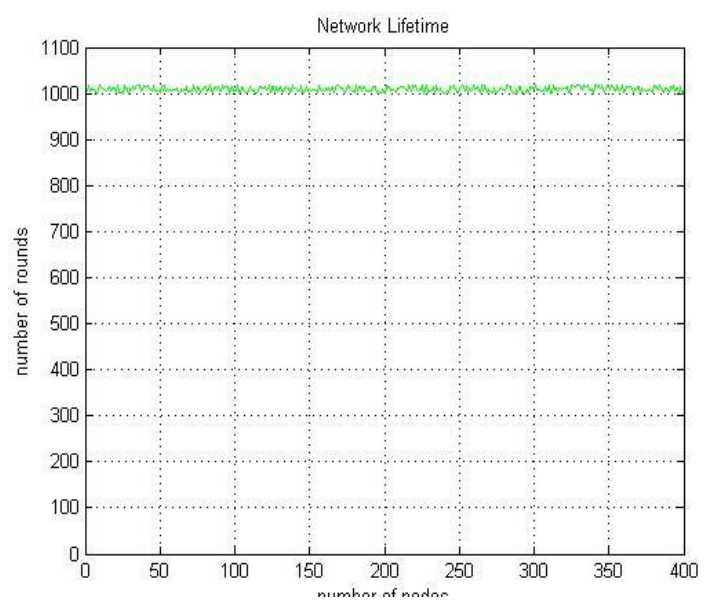

Fig 4:Lifetime of Network(rounds w.r.t nodes) 
The number of rounds that can be transmitted by implementing the algorithm proposed approach is 1000 iterations of data transfer for 400 nodes, which is significantly more than conventional pre existing methods [1].

\section{CONCLUSION}

After observation and analysis of the above results, it can be seen that the approach and system proposed performs better than the previously existing methods. This can be due to the fact that the proposed methodology puts together the features of adaptive clustering, cluster head selection intertwined with a threshold based approach that aids in reducing the energy consumption as well as transmissions and the number of the total transmissions that take place This proposed method can be applied to both heterogeneous and non heterogeneous networks that considerably enhances its usability and applicability of the proposed approach. The system proposed achieves higher network lifetime and also provides security as there is provision of re transmission of data after the delay time exceeded and if the data doesn't reach the control station. A comparative analysis can be carried out with the grid based routing algorithm proposed in [1] that acquires a maximum of 800 rounds of transmission for 400 sensor nodes in the network.

\section{REFERENCES}

[1] Abdul Waheed Khan, Abdul Abdullah, Mohammad Razzaque, Javed Bangash "VGDRA: A Virtual GridBased Dynamic Routes Adjustment Scheme for Mobile Sink-Based Wireless Sensor Networks, IEEE 2015

[2] Jun Wang, Xuegang Zhu1, Yong Cheng and Yongsheng Zhu, "A Distributed, Hybrid Energy-Efficient Clustering Protocol for Heterogeneous Wireless Sensor Network", International Journal of Grid and Distributed Computing, Vol. 6, No. 4, August, 2013.

[3] Zhanyang Xu, Yue Yin, Jin Wang, "An Energy-efficient Clustering Routing Algorithm for Heterogeneous
Wireless Sensor Networks", NGCIT 2013, ASTL Vol. 27,2013.

[4] Jun Wang, Xuegang Zhu1, Yong Cheng and Yongsheng Zhu, "A Distributed, Hybrid Energy-Efficient Clustering Protocol for Heterogeneous Wireless Sensor Network", International Journal of Grid and Distributed Computing, Vol. 6, No. 4, August, 2013

[5] B. S Taruna, Sakshi Shringi, "Zone-Based Clustering Protocol for Heterogeneous Wireless Sensor Networks", IJERA, 2013. S Taruna, Sakshi Shringi, "Zone-Based Clustering Protocol for Heterogeneous Wireless Sensor Networks", IJERA, 2013.

[6] Nilima Rani Das, Debashree Mishra Sar, "Analytical Study on Energy Efficient Clustering Algorithms for Wireless Sensor Networks", International Journal of Emerging Trends \& Technology in Computer Science, 2012.

[7] M.Jagadeeswara Reddy, P.Chenna Reddy, A.Ganesh, "Energy-Efficient Self Reconfigurable Clustering Approach for Heterogeneous Wireless Sensor Networks, International Journal of Computer Applications, 2012.

[8] Afroz Mansoori, "Particle Swarm Optimized Distributed Energy Efficient Clustering Protocol for WSN", International Journal of Digital Application \& Contemporary research, 2012.

[9] S.R.Boselin Prabhu, Assistant Professor, "A Survey of Adaptive Distributed Clustering Algorithms for Wireless Sensor Networks", International Journal of Computer Science \& Engineering Survey (IJCSES) Vol.2, No.4, November 2011.

[10] D. Kumar, T.C. Aseri, R.B. Patel, "EECDA: Energy Efficient Clustering and Data Aggregation Protocol for Heterogeneous", Int. J. of Computers, Communications \& Control, 2011. 